\title{
Effects of Idebenone (CV-2619) on the Concentrations of Acetylcholine and Choline in Various Brain Regions of Rats with Cerebral Ischemia
}

\author{
Mitsuru KAKIHANA, Naoki YAMAZAKI and Akinobu NAGAOKA \\ Biology Laboratories, Central Research Division, Takeda Chemical Industries, Ltd., \\ 17-85, Jusohonmachi 2-chome, Yodogawa-ku, Osaka 532, Japan \\ Accepted July 19, 1984
}

\begin{abstract}
The concentrations of acetylcholine (Ach) and choline in various brain regions of rats with and without cerebral ischemia and the effects of 6 - (10-hydroxydecyl)-2.3-dimethoxy-5-methyl-1,4-benzoquinone (idebenone, CV-2619) on the levels of these parameters were investigated. Cerebral ischemia was induced by a 200-sec occlusion of both common carotid arteries in animals in which both vertebral arteries had been permanently cauterized. The concentrations of Ach and choline in the brain were determined by means of pyrolysis gas chromatography. In normal rats, CV-2619 (10, 30 and $100 \mathrm{mg} / \mathrm{kg}$, i.p.) did not alter the concentrations of Ach and choline in the brain regions examined. In the ischemic rats, a significant decrease in Ach and a marked increase in choline were observed in the cerebral cortex, hippocampus, striatum, and diencephalon. A slight increase in the concentration of choline was also observed in the cerebellum and brain stem. Pretreatment with CV-2619 (10 mg/kg, i.p.) inhibited the decrease in Ach and the increase in choline in the forebrain regions. Moreover, the same dose of CV-2619 inhibited the increment of lactate content and tended to inhibit the decrement of ATP content in the cerebral cortex. These results indicate that CV-2619 inhibits alterations of the concentrations of Ach and choline in the brain of the ischemic rats: this inhibition may be due to the ameliorating effect of CV-2619 on the disturbance of cerebral energy metabolism under ischemic conditions.
\end{abstract}

In a previous report (1), we showed that CV-2619, 6-(10-hydroxydecyl)-2,3-dimethoxy-5-methyl-1,4-benzoquinone, has both protective and reversal effects on the amnestic performance deficits induced by a 200-sec cerebral ischemia immediately after one-trial passive avoidance learning. However, the mechanism by which CV-2619 produced these effects is not clear. Pharmacological experiments with humans and monkeys demonstrated that decreased cholinergic transmission in the central nervous system (CNS) resulted in impaired memory $(2,3)$. In addition, postmortem studies have revealed consistent and dramatic loss of hippocampal and cortical cholinergic pathways in patients with senile dementia (4-6). These studies strongly suggest a key role for central cholinergic pathways in memory. Furthermore, Gibson et al. (7) and Gibson and Duffy (8) have reported that mild hypoxia, in which the supply of energy to the brain is not impaired, reduced the synthesis of Ach in the CNS. In the present experiment, we attempted to study a possible mechanism by which CV-2619 protects against the amnesia induced by a 200-sec cerebral ischemia. Alterations of the concentrations of Ach, choline and energy metabolites in the brain of rats after a 200-sec cerebral ischemia and the effects of pretreatment with CV-2619 on these changes were determined.

\section{Materials and Methods}

Animals: Male Wistar rats, 9 weeks old, were purchased from Japan Clea Co. and 
maintained in our laboratories under controlled conditions of temperature $\left(23-24^{\circ} \mathrm{C}\right)$. humidity (50-60\%), and light (7:00-19:00).

Drugs: CV-2619 synthesized in this Central Res. Div. was suspended in 5\% arabic gum saline solution and injected intraperitoneally.

Preparations of cerebral ischemic rats: Cerebral ischemia was induced by the method of Pulsinelli and Brierley (9). Briefly, under pentobarbital anesthesia, the bilateral vertebral arteries were cauterized by a bipolar coagulator (Mizuhoika Kogyo, Micro-1D). and silk threads were placed around both common carotid arteries without interrupting carotid blood flow. On the next day, the rats were restrained by hand, subjected to bilateral common carotid artery occlusion (BCAO) by silk threads, and killed $200 \mathrm{sec}$ later by microwave irradiation ( $5 \mathrm{~kW}$ for $1.5 \mathrm{sec}$ ) (Toshiba, TMW-6402). These procedures were carried out in the morning to eliminate fluctuations of Ach and choline due to diurnal variation (10).

Determination of Ach and choline: The concentrations of Ach and choline in the brain were determined by the method of Maruyama et al. (11). Brain homogenates were prepared in $1.5-3.0 \mathrm{ml}$ of $1 \mathrm{~N}$ formic acid in acetone with $30 / \mathrm{l}$ of $0.3 \mathrm{mM}$ butyrylcholine iodide as an internal standard. After they stood in ice for $30 \mathrm{~min}$, the homogenates were centrifuged (Beckman. L8-55 Ultracentrifuge) at $20.000 \mathrm{rpm}$ and $4^{\circ} \mathrm{C}$ for $15 \mathrm{~min}$. The supernatant solutions were decanted and extracted with 2-4 $\mathrm{ml}$ of diethyl ether. After the ether was discarded. the aqueous portions were dried using a test tube concentrator (Taiyo Kagaku Kogyo. TC-8F). Three hundred microliters of acetonitrile was added to each tube, the residue in the tubes was broken up with a small glass rod, and 300 "l of propionyl chloride was added. These solutions were allowed to stand at $60^{\circ} \mathrm{C}$ for $40 \mathrm{~min}$ and then evaporated with a nitrogen stream. The residues were dissolved in 200 " 1 of distilled water, and the resultant solutions were transferred to small conical centrifuge tubes. Twenty microliters of potassium periodide solution was added to each sample and mixed well on a mixer. After centrifugation in a Eppendorf model
5412 centrifuge for $5 \mathrm{~min}$, the supernatants were removed, and the precipitates were dissolved in $50 \mu \mathrm{l}$ of acetonitrile. To remove excess iodine, approximately $5 \mathrm{mg}$ of $\mathrm{AG} 1$ $\times 8-\mathrm{Cl}^{-}$(Bio-Rad) was added, and the mixtures were shaken for $15 \mathrm{sec}$. Ten / $\iota$ portions were analyzed by gas chromatography (GC)

The samples were applied to the platinum filament of a pyrolyzer (Kotaki Shoji. PYRMS) and pyrolyzed at 2 A for $7.5 \mathrm{sec}$. GC analyses were carried out on a Shimadzu model GC-7A chromatograph. The GC was equipped with a glass column, $1.1 \mathrm{~m} \times 3.2 \mathrm{~mm}$ I.D., packed with 5\% OV-101, 5\% Jenden phase on 100-120 mesh Gaschrom O (Applied Science Div.) The carrier gas was nitrogen and the flow rate was $30 \mathrm{ml} / \mathrm{min}$. The column temperature was $100^{\circ} \mathrm{C}$ and the injector was maintained at $160^{\circ} \mathrm{C}$. For flame ionization, the detector was operated at $160^{\circ} \mathrm{C}$ and an air flow rate of $500 \mathrm{ml} / \mathrm{min}$ and a hydrogen flow rate of $50 \mathrm{ml} / \mathrm{min}$.

Determination of ATP and lactate: Brain tissues were homogenized with $4 \mathrm{ml}$ of $0.3 \mathrm{~N}$ perchloric acid. The homogenates, kept at $0^{\circ} \mathrm{C}$ to $4{ }^{\circ} \mathrm{C}$, were centrifuged and neutralized with $1.5 \mathrm{~N}$ potassium carbonate at $\mathrm{pH} 6.0$ 7.0. ATP and lactate were analyzed by the specific enzymatic fluorometric techniques of Lowry et al. (12) and Passonneau (13) as previously reported by Folbergrová et al. (14). Proteins were estimated using the Bio-Rad Protein Assay.

Effects of CV-2619 in intact rats: Wistar rats, 10-12 weeks old, were used. CV-2619 $(10,30$ and $100 \mathrm{mg} / \mathrm{kg}$ ) and $5 \%$ arabic gum saline solution $(2 \mathrm{ml} / \mathrm{kg}$ ) were given $30 \mathrm{~min}$ before microwave irradiation.

Alterations of Ach and choline levels after ischemia: Wistar rats, 10 weeks old, were used. The concentrations of Ach and choline in rats with cerebral ischemia were compared with those in sham operated rats and rats with both carotid artery occlusion but with intact vertebral arteries (BCAO rats). The rats were killed by microwave irradiation 200 sec after BCAO or sham operation.

Effects of CV-2619 in cerebral ischemic rats: Wistar rats, 10 weeks old, were used. $\mathrm{CV}-2619$ at a dose of $10 \mathrm{mg} / \mathrm{kg}$. which has been shown to have the most pronounced 
effect of memory improvement (1), were given $30 \mathrm{~min}$ before $\mathrm{BCAO}$. The rats were exposed to microwave irradiation $200 \mathrm{sec}$ after BCAO. BCAO rats were used as controls.

Statistical analysis: Statistical differences were evaluated using one-way analysis of variance with a posteriori comparisons by Tukey's test. All values were expressed as the mean \pm S.E.M.

\section{Results}

Effects of CV-2619 in intact rats: In intact rats, the concentrations of Ach in the cerebral cortex, hippocampus, striatum. diencephalon, cerebellum, and brain stem were $22.0 \pm 0.7,30.9 \pm 1.4,65.0 \pm 2.7,34.1 \pm$ $0.5, \quad 7.5 \pm 0.5$, and $31.5 \pm 0.9 \mathrm{nmol} / \mathrm{g}$ wet tissue, respectively; the corresponding concentrations of choline were $19.8 \pm 0.6$, $25.3 \pm 1.0, \quad 31.6 \pm 1.7, \quad 22.7 \pm 0.9, \quad 22.5 \pm 0.8$, and $20.5 \pm 0.7 \mathrm{nmol} / \mathrm{g}$ wet tissue. As shown in Table 1. CV-2619 (10,30 and $100 \mathrm{mg} / \mathrm{kg}$. i.p.) given $30 \mathrm{~min}$ before microwave irradiation had no effect on the concentrations of Ach and choline in these brain regions.

Alterations of Ach and choline levels after ischemia: The concentrations of Ach and choline in the brain of rats with a 200-sec cerebral ischemia were compared with those of sham rats and BCAO rats (Fig. 1). No neurological signs were observed in either the sham or BCAO rats, and no difference in Ach and choline levels between the two control groups was detected in any brain region. During the $200-\mathrm{sec}$ cerebral ischemia. all the rats in the ischemic group lost the righting reflex. The concentration of Ach was significantly decreased and that of choline markedly increased in the cerebral cortex. hippocampus, striatum, and diencephalon in the rats with cerebral ischemia. A slight increase in choline in the cerebellum and brain stem was also observed.

Effects of CV-2619 in cerebral ischemic rats: Figure 2 shows the effects of pretreatment with CV-2619 on the alterations of Ach and choline concentrations in the rats with cerebral ischemia. Changes similar to those in Fig. 1 occurred in the concentrations of Ach and choline in the various brain regions after the 200-sec cerebral ischemia (compare black column in Fig. 1 with striped column in Fig. 2). Pretreatment with CV-2619 (10 $\mathrm{mg} / \mathrm{kg}$, i.p.) attenuated the decrement of

Table 1. Effects of CV-2619 on the levels of Ach and choline in various brain regions of normal rats (nmol/g wet weight)

\begin{tabular}{|c|c|c|c|c|}
\hline & \multirow{2}{*}{$\begin{array}{c}5 \% \text { Arabic } \\
\text { gum }\end{array}$} & \multicolumn{3}{|c|}{$\mathrm{CV}-2619(\mathrm{mg} / \mathrm{kg})$} \\
\hline & & 10 & 30 & 100 \\
\hline \multicolumn{5}{|l|}{ Cerebral cortex } \\
\hline Ach & $22.0 \pm 0.7$ & $21.6 \pm 0.4$ & $20.9 \pm 0.5$ & $21.4 \pm 0.9$ \\
\hline Choline & $19.8 \pm 0.6$ & $19.4 \pm 0.6$ & $20.7 \pm 0.5$ & $21.6 \pm 0.9$ \\
\hline \multicolumn{5}{|l|}{ Hippocampus } \\
\hline Ach & $30.9 \pm 1.4$ & $31.0 \pm 1.0$ & $30.4 \pm 1.1$ & $27.1 \pm 1.4$ \\
\hline Choline & $25.3 \pm 1.0$ & $28.9 \pm 1.7$ & $29.2 \pm 1.7$ & $25.6 \pm 1.0$ \\
\hline \multicolumn{5}{|l|}{ Striatum } \\
\hline Ach & $65.0 \pm 2.7$ & $65.5 \pm 2.5$ & $65.6 \pm 2.0$ & $64.2 \pm 3.1$ \\
\hline Choline & $31.6 \pm 1.7$ & $36.5 \pm 2.5$ & $39.2 \pm 2.5$ & $36.8 \pm 3.8$ \\
\hline \multicolumn{5}{|l|}{ Diencephalon } \\
\hline Ach & $34.1 \pm 0.5$ & $33.2 \pm 0.8$ & $34.4 \pm 0.2$ & $33.6 \pm 0.9$ \\
\hline Choline & $22.7 \pm 0.9$ & $25.8 \pm 1.5$ & $26.6 \pm 1.3$ & $25.4 \pm 2.3$ \\
\hline \multicolumn{5}{|l|}{ Cerebellum } \\
\hline Ach & $7.5 \pm 0.5$ & $7.2 \pm 0.4$ & $6.9 \pm 0.4$ & $7.2 \pm 0.2$ \\
\hline Choline & $22.5 \pm 0.8$ & $23.2 \pm 1.2$ & $25.2 \pm 1.3$ & $26.6 \pm 1.2$ \\
\hline \multicolumn{5}{|l|}{ Brain stem } \\
\hline Ach & $31.5 \pm 0.9$ & $31.2 \pm 0.6$ & $30.9 \pm 0.5$ & $31.5 \pm 1.1$ \\
\hline Choline & $20.5 \pm 0.7$ & $20.5 \pm 0.9$ & $20.8 \pm 0.5$ & $23.2 \pm 0.9$ \\
\hline
\end{tabular}

CV-2619 was given i.p. 30 min before microwave irradiation. All values are the mean \pm S. E.M. of 7-8 rats. 


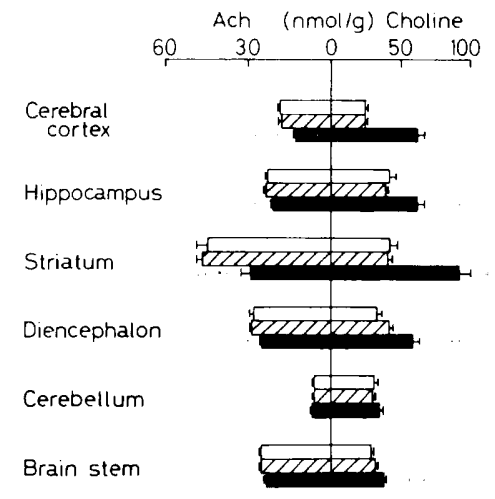

Fig. 1. Changes in Ach and choline contents in various brain regior,s $200 \mathrm{sec}$ after bilateral carotid artery occlusion in rats with and without permanent electrocauterization of the vertebral artery bilaterally. Values are the mean $\$$ S.E.M. White column: sham operated rats $(N=6)$. Striped column: rats with bilateral carotid artery occlusion $(N=9)$. Black column: cerebral ischemic rats $(N=9)$. ${ }^{*} P<0.05$. ${ }^{*} P<0.01$ vs. sham operated rats. $+P<005$, $++P<0.01$ vs. rats with bilateral carotid artery occlusion.

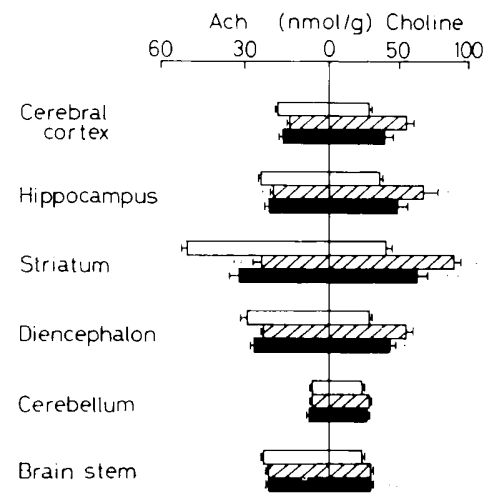

Fig. 2. Effect of CV-2619 on Ach and choline contents in various brain regions of rats with a 200-sec cerebral ischemia. Values are the mean \pm S.E.M. CV $-2619(10 \mathrm{mg} / \mathrm{kg})$ and $5 \%$ arabic gum $(2 \mathrm{ml} / \mathrm{kg}$ ) were given ip $30 \mathrm{~min}$ before the $200-\mathrm{sec}$ cerebral ischemia. White column: rats with bilateral carotid artery occlusion injected with 5\% arabic gum $(N=7)$. Striped column: cerebral ischemic rats injected with $5 \%$ arabic gum $(N=6)$. Black column: cerebral ischemic rats treated with $\mathrm{CV}-2619$ $(\mathrm{N}=8) .{ }^{*} \mathrm{P}<0.05,{ }^{*} \mathrm{P}<0.01$ vs, bilateral carotid artery occluded rats. ${ }^{+} P<0.05$ vs. cerebral ischemic rats injected with $5 \%$ arabic gum.

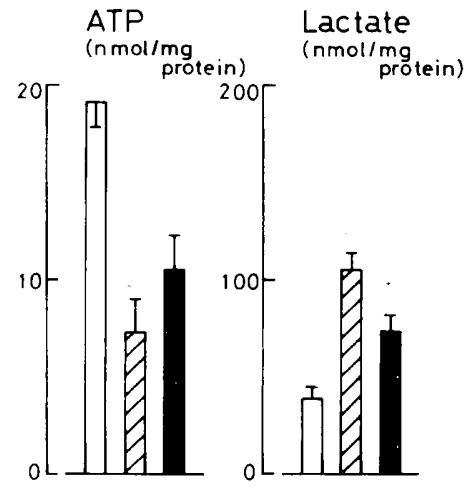

Fig. 3. Effect of CV-2619 on ATP and lactate contents in the cerebral cortex of rats with the $200-\mathrm{sec}$ cerebral ischemia. Values are the mean \pm S.E.M. CV-2619 $(10 \mathrm{mg} / \mathrm{kg})$ and $5 \%$ arabic gum (2 ml/kg) were given i.p. $30 \mathrm{~min}$ before the $200-\mathrm{sec}$ cerebral ischemia. White column: rats with bilateral carotid artery occlusion injected with 5\% arabic gum $(N=8)$. Striped column: ischemic rats injected with $5 \%$ arabic gum $(N=13)$. Black columri: cerebral ischemic rats treated with $C V-2619 \quad(N=13)$. ${ }^{*} P<0.05,{ }^{* *} P<0.01$ vs. bilateral carotid artery occluded rats. $+P<0.05$ vs. cerebral ischemic rats injected with $5 \%$ arabic gum.

Ach and the increment of choline in the forebrain of the ischemic rats: the increase in choline was significantly inhibited in the cerebral cortex, striatum, and diencephalon. The changes in Ach in the forebrain and in choline in the hippocampus tended to be attenuated.

In the last experiment, the effects of $\mathrm{CV}$ 2619 on the changes in ATP and lactate were assessed in the cerebral cortex of the ischemic rats (Fig. 3). The cerebral ischemia caused a marked decrease in ATP and increase in lactate content. Pretreatment with CV-2619 prevented the increase in lactate and tended to attenuate the decrease in ATP.

\section{Discussion}

The great sensitivity of the brain to a decrease in oxygen (hypoxia or ischemia) has been well established. Memory and the ability to learn complex tasks in man are impaired when arterial oxygen tension is decreased to about $50 \mathrm{mmHg}$ from a normal level of approximately $100 \mathrm{mmHg}$ (15). The molecular basis of this sensitivity is unknown. 
The effects of hypoxia are not due to a measurable decrease in the levels of energy metabolite (e.g., ATP) or the adenylate energy charge (16-18). Gibson et al. (7) and Gibson and Duffy (8) have reported that in mild hypoxia, the levels of ATP and the adenylate energy charge in the brain were normal, but the synthesis of Ach was markedly reduced. They proposed that impairment of memory and learning ability during hypoxia in man may be due to the disruption of central cholinergic systems. In a previous report (1), we showed that cerebral ischemia for 200-600 sec after the acquisition of one-trial passive avoidance learning resulted in the induction of retrograde amnesia in rats; physostigmine $(0.1$ and $0.2 \mathrm{mg} / \mathrm{kg}$, i.p.) given before the retention test significantly reversed the amnesia induced by the 200-sec cerebral ischemia immediately after the avoidance learning. In the present experiment, the 200sec cerebral ischemia caused a decrease in Ach and an increase in choline in the forebrain. The results from these two sets of experiments indicate that central cholinergic dysfunction may be responsible for the development of the amnesia induced by the 200-sec cerebral ischemia. According to Take et al. (19), the concentrations of norepinephrine, dopamine, and serotonin in various brain regions of rats with a 10 -min cerebral ischemia were unchanged.

Our previous experiment (1) also demonstrated that a novel compound, CV-2619, improved the amnestic performance deficits induced by the cerebral ischemia. The effects of CV-2619 were observed when the drug was given before and after the 200-sec ischemia and before the retention test. The dose-response effect of CV-2619 on the improvement of the amnesia varied slightly with the timing of administration. A linear dose-response relationship was detected when the compound was given before the retention test, and a bell-shaped one was obtained when it was given before and after the ischemia. In the present experiment, we studied the effect of pre-ischemia treatment with CV-2619 on the alterations in the levels of brain Ach and choline in rats with cerebral ischemia to investigate a possible mechanism of the anti-amnestic action of CV-2619. CV-2619 at a dose of $10 \mathrm{mg} / \mathrm{kg}$ was administered $30 \mathrm{~min}$ before the induction of the cerebral ischemia, because the most pronounced effect of memory improvement had been shown in this dose (1) and the highest concentration of CV-2619 in the blood had been observed 15-30 min after systemic administration ( $\mathrm{H}$. Torii et al., unpublished data). Pretreatment with $\mathrm{CV}$ 2619 tended to inhibit the decrease in Ach in the forebrain and the increase in choline in the hippocampus, and it significantly inhibited the increase in choline in the cerebral cortex, striatum, and diencephalon. This result suggests that the protective effect of CV-2619 on the ischemia inducedamnesia may be due, at least in part, to the amelioration of the disruption of central cholinergic systems.

A growing body of clinical and experimental data (2-6) has revealed that cortical and hippocampal cholinergic pathways play a very important role in the formation of memory. In the present study, a decrease in Ach and an increase in choline content were observed in the striatum and diencephalon as well as in the cerebral cortex and hippocampus. It is not clear, however, that the disruption of cholinergic neurons in the striatum and diencephalon may be responsible for the amnesia induced by the cerebral ischemia.

The protective effects of CV-2619 on experimentally induced cerebral ischemia have been also studied in stroke-prone spontaneously hypertensive rats (SHRSP). In SHRSP with both common carotid artery occlusion, neurological signs followed a marked reduction in the cerebral blood flow (CBF) and a decrease in the concentration of ATP in the brain $(20,21)$. Pretreatment with CV-2619 had no effect on the CBF, but attenuated the decrease in ATP and delayed the onset-time of neurological signs (in preparation). These observations led us to study the effect of CV-2619 on cerebral energy metabolism in the 200-sec cerebral ischemic rats: the effects of CV-2619 on the alterations of the concentrations of ATP and lactate were examined in the cerebral cortex. Pretreatment with CV-2619 inhibited or 
tended to inhibit the changes in the levels of these parameters. This result suggests that CV-2619 primarily ameliorates the disruption of cerebral energy metabolism and consequently inhibits central cholinergic dysfunction. Further studies including the turnover rate of central cholinergic neurons are necessary to investigate the mechanisms in more detail.

It is well known that the concentration of choline is markedly increased in the brain after cerebral ischemia as shown in the present experiment. Choline is not only a constituent of the transmitter Ach but is also a basic structural element of the neurons in the form of phospholipids. Dross and Kewitz (22) and Dross (23) reported that the postmortem accumulation of choline is due to an enzymic process that liberates choline. primarily glycerophosphorylcholine and hence phosphatidylcholine. Other components of phospholipids, fatty acids, also accumulate in the brain after ischemia, resulting in the increase of so-called free fatty acids (FFAs) (24). Of the major FFAs, arachidonic and stearic acids arise primarily from membrane lipids, which is consistent with the reduction in the brain phospholipids during hypoxia (25) and ischemia (26). Membrane phospholipids are strongly implicated in synaptic transmission (27, 28) and phospholipid metabolism dysfunction could impair synaptic transmission (29, 30). In addition. it has been reported that fatty acids inhibit oxidative phosphorylation of in vitro mitochondrial preparations (31), and arachidonic acid has been shown to be capable of inducing brain edema in vitro (32) and in vivo (33). Because CV-2619 markedly attenuated the increment of choline in cerebral tissues, we speculate that CV-2619 may inhibit the occurrence of dysfunction in the neuronal membrane.

In summary, pretreatment with CV-2619 inhibited changes of a neurotransmitter, Ach. and a metabolite of Ach and phospholipids, choline, in the brain of rats with 200-sec cerebral ischemia; this action may be responsible for beneficial effects of $\mathrm{CV}-2619$ on memory impairmemt induced by the ischemia.

Acknowledgments: The authors would like to thank Dr. J.R. Miller for his helpful comments and Mr. K. Ikeda and Mrs. E. Fujiwara for their excellent technical assistanice.

\section{References}

1 Yamazaki, N., Take, Y., Nagaoka, A. and Nagawa, $Y$.: Beneficial effect of idebenone (CV-2619) on cerebral ischemia-induced amnesia in rats. Japan. J. Pharmacol. 36, 349-356 (1984)

2 Drachman, D.A. and Leavitt, J.: Human memory and the cholinergic system: A relationship to aging? Arch. Neurol. 30, 113-121 (1974)

3 Bartus, R.T. and Johnson, H.R.: Short-term memory in the rhesus monkey: Disruption from the anticholinergic scopolamine. Pharmacol. Biochem. Behav. 5, 39-46 (1976)

4 Davies, P. and Maloney, A.J.F.: Selective loss of central cholinergic neurons in Alzheimer's disease. Lancet 2, 1403 (1976)

5 Perry, E.K., Perry, L.H., Biessed, G. and Tomlinson, B.E.: Necropsy evidence of central cholinergic deficits in senile dementia. Lancet 1, 189 (1977)

6 Coyle, J.J., Price, D.L. and Delong, M.R.: Alzheimer's disease: A disorder of cortical cholinergic innervation. Science 219, 11841190 (1983)

7 Gibson, G.E., Shimada, M. and Blass, J.P.: Alterations in acetylcholine synthesis and cyclic nucleotides in mild cerebral hypoxia. J. Neurochem. 31, 759-760 (1978)

8 Gibson, G.E. and Duffy, T.E.: Impaired synthesis of acetylcholine by mild hypoxic hypoxia or nitrous oxide. J. Neurochem. 36, 28--33 (1981)

9 Pulsinelli, W.A. and Brierley, J.B.: A new model of bilateral hemispheric ischemia in the unanesthetized rat. Stroke 10, 267-272 (1979)

10 Hanin, I., Massarelli, R. and Costa, E.: Acety!choline concentrations in rats brain: Diurnal oscillation. Science 170, 341-342 (1970)

11 Maruyama, Y., Kusaka, M., Mori, J., Horikawa, A. and Hasegawa, Y.: Simple method for the determination of choline and acetylcholine by pyrolysis gas chromatography. J. Chromatography 14, 121-127 (1979)

12 Lowry, O.H., Passonneau, J.V., Hasselberger, F.X. and Schulz, D.W.: Effect of ischemia on known substrates and cofactors of the glycolytic pathway in brain. J. Biol. Chem. 239, 18-30 (1964)

13 Passonneau, J.V.: L-(+)-lactate determination with lactate dehydrogenase and NAD: fluorometric method. In Methods of Enzymatic Arralysis, Edited by Bergmeyer, H.U., Vol. III, p. 1468-1472. Academic Press, New York 
(1974)

14 Folbergrová J., MacMillan, V. and Siesjö, B.K.: The effect of moderate arid marked hypercapnia upon the energy state and upon NADH/NAD+ ratio of the rat brain. J. Neurochem. 19, 24972505 (1972)

15 Luft, U.C.: Aviation physiology-the effect of altitude. In Handbook of Physiology. Edited by Fenr, W.O. and Rahn, H., Vol. II, p. 1099-1145. American Physiological Society, Washington. D.C. (1965)

16 Duffy, T.E., Nelson, S.R. and Lowry, O.H.: Cerebral carbohydrate metabolism during acute hypoxia and recovery. J. Neurochem. 19, 959977 (1972)

17 Bachelard, H.S., Lewis, L.D., Ponten, U. and Siesjö, B.K.: Mechanisms activating glycolysis in the brain in arterial hypoxia. J. Neurochem. 22, 395-401 (1974)

18 Gibson, G.E. and Blass, J.P.: Impaired synthesis of acetylcholine in brain accompanying mild hypoxia and hypoglycemia. J. Neurochem. 27, 37-42 (1976)

19 Take, Y., Narumi, S., Nagai, Y., Kurihara, E., Saji, Y. and Nagawa. Y.: Neurochemical study of the temporary cerebral ischemic rats produced by bilateral vertebral and carotid artery occlusion. Folia Pharmacol. Japon. (in press)

20 Kakihana, M., Suno, M., Shibota, M., Fujiwara, K., Hamajo, K. and Nagaoka, A.: Changes of behavior, cerebral blood flow and metabolism after bilateral carotid artery occlusion ir strokeprone SHR, stroke-resistant SHR and normal Wistar-Kyoto rats. Japan. Heart J. 21, 569 (1980)

21 Suno, M., Kakihana, M., Shibota, M. and Nagaoka, A.: Mechanism of increased sensitivity to cerebral ischemia following carotid artery occlusion in stroke-prone spontaneously hypertensive rats: Importance of genetic factor. Stroke 12, 246-250 (1981)

22 Dross, K. and Kewitz, H.: Concentration and origin of choline in the rat brain. Naunyn Schmiedebergs Arch. Pharmacol. 274, 91-106 (1972)
23 Dross, K.: Effects of di-isopropylfluorophosphate on the metabolism of choline and phosphatidylcholine in rat brain. J. Neurochem. 24, 701706 (1975)

24 Bazan, N.G., Jr.: Effects of ischemia and electroconvulsive shock on free fatty acid pool in the brain. Biochim. Biophys. Acta 218, 1-10 (1970)

25 Yatsu, F.M. and Moss, S.A.: Brain lipid changes following hypoxia. Stroke 2, 587-593 (1971)

26 Rodriguez de Turco, E.B., Cascone, G.D., Pediconi, M.F. and Bazan, N.G.: Phosphatidate, phosphatdylinositol, diacylglycerols, and free fatty acids in the brain followirig electroshock. anoxia, or ischemia. In International Symposium on Function and Biosynthesis of Lipids, Edited by Bazan, N.G., Brenner, R.R. and Guisto, N.M., p. 389-396, Plenum Press, New York (1977)

27 Hokin-Neaverson, M.: Metabolism and role of phosphatidylinositol in acetylcholine stimulated membrane function. Adv. Exp. Med. Biol. 83, 429-446 (1976)

28 Hawthorne, J.N. and Pickard, M.R.: Phospholipids in synaptic function. J. Neurochem. 32, 5-14 (1979)

29 Shiu, G.K. and Nemoto, E.M.: Barbiturate attenuation of brain free fatty acid liberation during global ischemia. J. Neurochem. 37, 1448-1456 (1981)

30 Yoshida, S., Inoh, S., Asano, T., Sano, K., Shimasaki, H. and Ueta, N.: Brain free fatty acids, edema, and mortality in gerbils subjected to transient, bilateral ischemia, and effect of barbiturate anesthesia. J. Neurochem. 40, 1278-1286 (1983)

31 Kuwashima, J., Fujitani, B., Nakamura, K., Kadokawa, T., Yoshida, K. and Shimizu, M.: Biochemical changes in unilateral brain injury in the rat: a possible role of free fatty acid accumulation. Brain Res. 110, 547-557 (1976)

32 Chan, P.H. and Fishman, R.A.: Brain edema: Induction in cortical slices by polyunsaturated fatty acid. Science 201, 358-360 (1978)

33 Prioleau, G.R., Fishman, R.A. and Chan, P.H.: Induction of brain edema by fatty acids in vivo. Ann. Neurol. 6, 156 (1979) 